\title{
The Control Strategy of Soft Landing Trajectory of Lunar Craft
}

\author{
Zhiyong Lin
}

North China Electric Power University. Baoding, Hebei, China

Keywords: Centroid-based Dynamic Model; Numerical Iteration Method; Parameterize Method.

\begin{abstract}
The purpose of this paper is study soft landing strategies of the lunar craft during landing on the moon. On the basis of the fixed selenocenter coordinate system, we set up a braking coordinate system in the main reduction stage. Build the centroid-based dynamic differential equation model to describe the relation among different variables and for the target of least fuel consumption. Use the parameterize method to keep complex unknown variables approximate for the power function. Try the numerical iteration method for solving the model. When it comes to an approximate optimal goal, we finally get result that main reduction stage take 585 seconds, and angle of thrust direction show increased over time, and thrust decreases with time, and the rate of reduction increases with time gradually.
\end{abstract}

\section{Introduction}

The moon landings is the dream of the mankind, at the same time, the development of the aerospace play an important role in a country's national defense and the development of science and technology. Guarantee the accuracy of the lunar craft to achieve a safe landing in the area of the moon, need reasonable landing trajectory and control strategy. Because of its load capacity is limited, the whole process needs to reduce the energy consumption, and reach the preset orbit in various stages of the state. Due to the surface of the moon has no atmosphere, the speed of the aircraft has to be completely offset by the engine braking. The soft landing stage can be divided into six stages, this paper discuss the main reduction stage. In this stage, aircraft relative speed from $1700 \mathrm{~m} / \mathrm{s}$ gradually dropped to $37 \mathrm{~m} / \mathrm{s}$, most of the energy cost in this stage, so we need to optimize landing orbit to reduce fuel consumption.

\section{2. centroid-based dynamics model}

\subsection{Description}

The main reduction in lunar craft is start $15 \mathrm{~km}$ high away from the moon perilune. In hundreds of seconds, It rapidly reduce velocity in the vertical direction and descend by $12 \mathrm{~km}$. In the process of the

downhill, the orbit of lunar craft is affected by gravity, momentum, and its quality. Landing orbit brake system is established in the longitudinal plane. And Centroid-based dynamics differential equation model is established aiming at the optimize target of least- costing consumption.

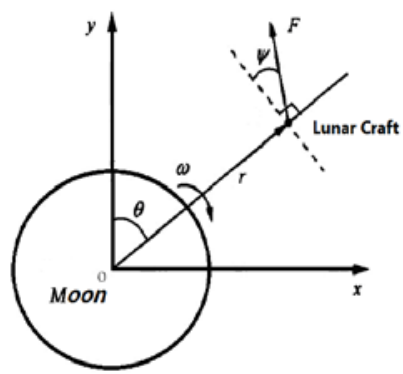

Figure 1 the landing orbit brake coordinates system

\subsection{Establish Centroid-based Dynamic Differential Equation}

Establish a landing orbit brake coordinates system in rail longitudinal landing plane. Set the month center as the origin of coordinates, $y$ axis pointing to the beginning of the main reducer braking point 
which is perilune, the $\mathrm{x}$ axis pointing in the direction that lunar craft began to descend, and its braking system is as follows (figure 1):

According to the minimum energy principle, under the condition of the speed, the relative displacement and so on, establishing a centroid dynamics differential equation model, which is described as below:

$$
\left\{\begin{array}{l}
\dot{r}=\zeta \\
\dot{\zeta}=(F / m) \sin \psi-\mu / r^{2}+r \omega^{2} \\
\dot{\theta}=\omega \\
\dot{\omega}=-[(F / m) \cos \psi+2 \zeta \omega] / r \\
\dot{m}=-F / i \\
\mu=4.9 \times 10^{6} \mathrm{~km}^{3} / \mathrm{s}^{2}, i=2940 \mathrm{~m} / \mathrm{s}
\end{array}\right.
$$

Where $r, \theta, \omega, m$ stand for the selenocentric distance, polar angle, angular velocity and quality. $\zeta$ is the velocity along the direction of $r . \mu$ is the moon's gravitational constant. $i$ is specific impulse. $\psi$ is engine thrust and local angle between the horizontal thrust direction Angle. The rest of the variable is a function of time $t$.

The key of the main reduction initial position is the perilune elliptical orbit. Make the initial time $t_{0}=0$. and trough the relevant information, determine the end time of main reduction is about $t_{f}=600$ s. The corresponding initial conditions are :

$$
\left\{\begin{array}{l}
r(0)=R+h \\
\zeta(0)=0 \\
w(0)=\sqrt{\frac{\mu}{r_{0}}} \\
t_{f}=600 \pm \Delta \\
\theta(0)=0
\end{array}\right.
$$

The boundary conditions are:

$$
\left\{\begin{array}{c}
r\left(t_{f}\right)=r_{f} \\
v\left(t_{f}\right)=v_{f} \\
\omega\left(t_{f}\right)=0
\end{array}\right.
$$

Thrust and orbital direction angle are:

$$
\left\{\begin{array}{l}
F_{\min } \leq F(t) \leq F_{\max } \\
\psi(0) \leq \psi(t) \leq \psi\left(t_{f}\right)
\end{array}\right.
$$


The objective function is:

$\min J=\int_{t_{0}}^{t_{f}} \dot{m} d t$

\subsection{Normalization}

In the process of orbit optimization, the order of every state variable is very different. In order to enhance the precision of model, we use normalization method. There are expressions:

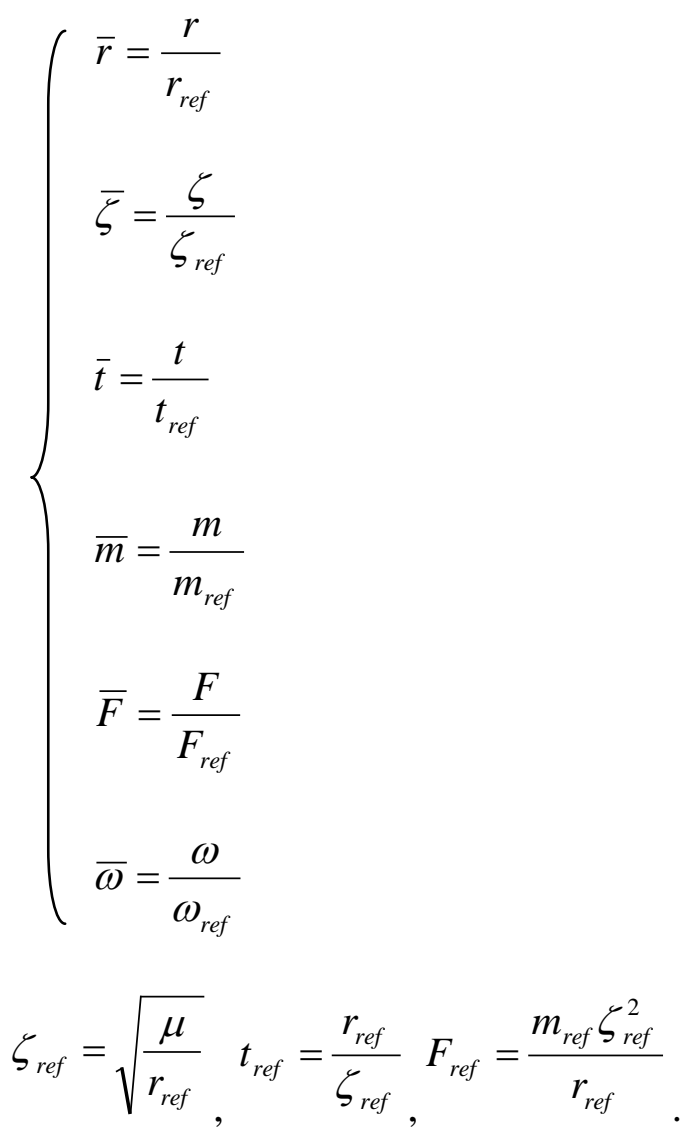

Then change formula (1) into fellows:

$$
\left\{\begin{array}{l}
\dot{\bar{r}}=\bar{\zeta} \\
\dot{\phi}=\bar{F} / \bar{m} \cdot \sin \varphi-\bar{r}^{-2}+\bar{r} \bar{\omega}^{2} \\
=\bar{F} /(\bar{m} \cdot \bar{r}) \cdot \cos \varphi+2 \bar{\zeta} \bar{\omega} \\
\dot{\bar{m}}=m_{0}+\bar{F} / i
\end{array}\right.
$$

$\min \bar{J}=\int_{t_{0}}^{t_{f}} \dot{\bar{m}} d \bar{t}$

\subsection{Simplify Differential Equation}

Change formula (1)to state equations as follows:

$\dot{x}=f(x, u)$

Where, $x=[\bar{r}, \bar{\theta}, \bar{\zeta}, \bar{\omega}, \bar{m}]^{T} u=[\bar{F}, \psi]^{T}$ 
How to find an appropriate guidance law to minimize the fuel consumption is our goal. That is how to find the direction and speed of the thrust to reduce the fuel consumption.

Initial condition are:

$$
\left\{\begin{array}{l}
\bar{r}(0)=0 \\
\bar{\zeta}(0)=0 \\
\bar{\omega}(0)=\omega_{0} / \sqrt{\frac{\mu}{r_{0}}} \\
t_{f}=600 \pm \Delta \\
\bar{\theta}(0)=0
\end{array}\right.
$$

\subsection{Simplify the direction of lunar landing trajectory}

The landing trajectory of satellite is similar to parabola. So we use parameterize methods to quantify it. Using the characteristics of the basis functions to approximate control variable function space, and turn the function space of infinite dimension parameters into the coefficient of basis functions. Power function has the characteristics of simple and orthogonalization, therefore three times power function was carried out on fitting the landing trajectory angle in this paper. The lunar soft landing trajectory is discrete. And we divided it into $n$ small pieces. Each piece is close to linear. Thrust direction parameter of the orbit, that is $\psi$, which can be only determined by every section.

And the every pieces of time is

$t_{i}=t_{0}+i \cdot\left(t_{n}-t_{0}\right) / n$

The function expression for the corresponding relationship between soft landing direction angle and time is:

$$
\psi(t)=\lambda_{0}+\lambda_{1} t+\lambda_{2} t^{2}+\lambda_{3} t^{3}
$$

So, in this way, when get the data of time and orbital direction angle, we can further fit them into formula (13). And we also can get the corresponding parameter values -- $\lambda_{i}$.

\subsection{Simplify the thrust}

In the process of landing, thrust mainly overcome the radial velocity caused by the moon's gravity .By formula (7), we derive as the follows:

$$
\bar{F} \propto-i \frac{d \bar{m}}{d t}
$$

By parameterize method, we use the power function for fitting expression of thrust to we simplify the formula. In the formula (10) we can know $\bar{F}$ is some formula that presenst decreasing momentum. So we adopt the secondary power function for fitting. Here it is:

$$
\bar{F}(t)=k_{0}+k_{1} t+k_{2} t^{2}
$$

\section{Solution of the model}

\subsection{Algorithm Design}

Because the values at the boundary conditions in (7) we do not know quite completely. It is difficult for us to exactly solve the problem. We settle for second best, for the sake of simplicity, we design an iterative algorithm. First we divide the time into pieces and then traverse them. So that we can achieve the optimal result. Under meeting the condition of precision.

\subsection{Steps of Numerical Iteration Method}


Setp 1: Due to the initial condition and part of the end value we've known, we assume thatwe've gain the fixed end parameter values. There are $r\left(t_{f}\right)=1737.36, \zeta\left(t_{f}\right)=57, \omega\left(t_{f}\right)=0$.

After using normalized, there we get new $\bar{r}\left(t_{f}\right), \bar{\zeta}\left(t_{f}\right)$ and $\bar{\omega}\left(t_{f}\right)$.

Step 2: Divide the small area $\Delta$ in formula (9) into $\mathrm{n}$ pieces. By fill in the corresponding experimental value, we work out $F(t)$ and $\psi(t)$. Let them be yhe variables and join into spanning space, that is $C=L(\psi, F)$. Within the $C$ space we set some value and then obtain the corresponding function results. Use the results to the objective function for searching the optimal value.

Step 3: Get $n \times n$ dimensional vector from the C. Quantitate the corresponding boundary condition calculated by every vector, and join the two parameters into one. Obviously boundary condition is the variable, so we can only ensure the initial condition. But when we calculate the problem every time, the Initial condition will produce the new condition to cover the old one. As a result, there comes new initial differential equation.

Step 4: Work out the initial value of differential equations (7) by the Runge-Kutta algorithm. Because we have a group of differential equations, so it is crucial to traverse everyone to solve the initial condition problem. Finally, we get a set of solutions. Set the accuracy like $\varepsilon$, when the solution last step reduce the result next step less than $\varepsilon$, finish the algorithm. When $t_{i}$ constantly tend to $t_{f}$, we always can find the optimal solution $\bar{J}$ in the process of exhaustive method.

\subsection{Results of the Model}

According to the assumping the corresponding data, we simply simulated the landing trajectory of lunar craft. Work out the parameter in formula (13), we get:

$$
\begin{aligned}
& \lambda_{0}=2.6670, \lambda_{1}=0.0165 \\
& \lambda_{2}=0.0001, \lambda_{3}=-2.361 \times 10^{-6}
\end{aligned}
$$

And also work out the parameter in formula (15):

$k_{0}=7500, k_{1}=0.521, k_{2}=-0.024$

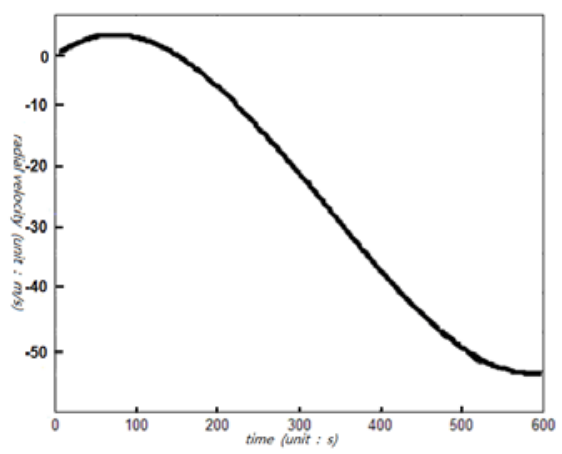

The results are as follows:

Figure 1 The radial velocity of the lunar craft changing with time (positive and negative value represents direction)

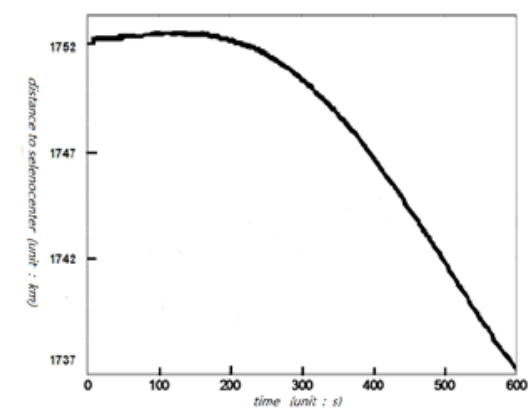

Figure 2 The distance that lunar craft landing into the moon with time

By calculation, we get the approximate solution that main reduction stage took 585 seconds to do. And the optimized consumption of fuel add up to $1371.5 \mathrm{~kg}$. 


\section{Conclusion}

The main drop process of Lunar craft from the perilune, falling to where $3 \mathrm{~km}$ from the ground, keeps changing its velocity and direction. According to the relationship between various physical quantities, differential mass center brake model is built combined with a variety of evaluation methods, which lead s to the simplification of corresponding model, and we work out the basic movements of the main drop trajectories. Contrast through a variety of literature, we found that the algorithm of this paper have certain robustness. But because of the approximation adopted in many places, there are certain errors in the way itself and which still needs a large space for improvement.

\section{References}

[1] Chuang C H, Goodson T D, Hanson J. Fuel 2 optimal, low 2 and medium 2 thrust or bit transfers in large number s of burns [ R ] . A IAA 9423650, 1994

[2] LIU Dun. Spacecraft Orbital Dynamics [M]. Harbin: Harbin Institute of Technology Press, 1991

[3] Pierson B L, Kluever C A. Three - stage approach to optimal low - thrust earth-moon trajectories [J]. J of Guidance, Control, and Dynamics, 1994, 17(6): 12751282.

[4] SHAN Yongzheng,DUAN Guangren,LV Shiliang. The optimal control of lunar probe soft landing [J]. Optics and Precision Engineering, 2009, 09:2153-2158.

[5] WANG Da-yi ,LI Tie-shou, MA Xing-rui. The optimal soft landing the moon two-point boundary value problems of numerical solution [J]. Aerospace Control, 2000, 03:44-49+55.

[6] WAN Jie,Cui Nai-gang,LIU Dun. Constant thrust lunar lander research the optimal soft landing orbit [J]. High-tech communications,2003, 13(4)

[7] YutakaTakano, Shigeaki Wada, Noriki Iwanaga. Study on reference trajectory for the Iunar Lander. NASDA TechnicaI Memorandum(NASDA-TMR-950013T), National Space Development Agency of Japan,1996

[8] ZHU Jianfeng,XU Shijie. Optimization of lunar soft landing orbit based on adaptive simulated annealing genetic algorithm [J]. Acta Aeronautica et Astronautica Sinica, 2007, 04:806-812 